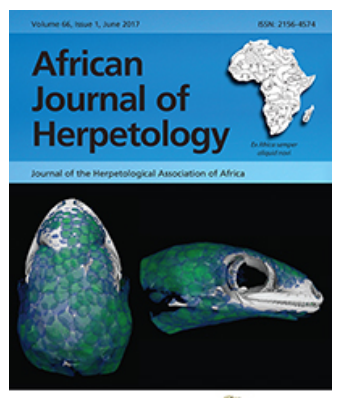

African Journal of Herpetology

\title{
Genetic diversity and differentiation of the Western Leopard Toad (Sclerophrys pantherina) based on mitochondrial and microsatellite markers
}

Jessica M. da Silva, Kevin A. Feldheim, G. John Measey, Stephen DoucetteRiise, Ryan J. Daniels, Lucas F. Chauke \& Krystal A. Tolley

To cite this article: Jessica M. da Silva , Kevin A. Feldheim, G. John Measey, Stephen Doucette-Riise, Ryan J. Daniels, Lucas F. Chauke \& Krystal A. Tolley (2017) Genetic diversity and differentiation of the Western Leopard Toad (Sclerophrys pantherina) based on mitochondrial and microsatellite markers, African Journal of Herpetology, 66:1, 25-38, DOI: 10.1080/21564574.2017.1294115

To link to this article: http://dx.doi.org/10.1080/21564574.2017.1294115

+ View supplementary material

Submit your article to this journal $₫$
Published online: 23 Jun 2017.

Wll Article views: 18 


\title{
Genetic diversity and differentiation of the Western Leopard Toad (Sclerophrys pantherina) based on mitochondrial and microsatellite markers
}

\author{
Jessica M. DA Silva ${ }^{1,2 *}$, KeVIn A. Feldheim ${ }^{3}$, \\ G. John Measey ${ }^{1,4}$, Stephen Doucette-Riise ${ }^{1,5}$, Ryan \\ J. Daniels ${ }^{1}$, Lucas F. Chauke ${ }^{1} \&$ Krystal A. Tolley ${ }^{1,2}$
}

\begin{abstract}
${ }^{1}$ Kirstenbosch Research Centre, South African National Biodiversity Institute, Private Bag X7, Claremont, Cape Town, South Africa, ${ }^{2}$ Department of Botany \& Zoology, University of Stellenbosch, Private Bag X1, Matieland 7602, Stellenbosch, South Africa, ${ }^{3}$ Pritzker Laboratory for Molecular Systematics and Evolution, The Field Museum, 1400 S. Lake Shore Drive, Chicago, IL 60605, USA; ${ }^{4}$ Centre for Invasion Biology, Department of Botany and Zoology, Stellenbosch University, Natural Sciences Building, Matieland, Stellenbosch, South Africa; ${ }^{5}$ Department of Biological Sciences, University of Cape Town, Private Bag X3, Rondebosch 770, Cape Town, South Africa
\end{abstract}

\begin{abstract}
Intraspecific genetic diversity provides the basis for evolutionary change and is therefore considered the most fundamental level of biodiversity. Mitochondrial DNA (mtDNA) and microsatellite loci are the markers most typically used in population-level studies; however, their patterns of genetic variation are not always congruent. This can result in different interpretations of the data, which can impact on management decisions, especially for threatened species. Consequently, in this study, we developed and analysed novel microsatellite markers for the Endangered Western Leopard Toad (WLT), Sclerophrys pantherina, and compared the results to previously published mtDNA data to compare the level of genetic diversity between the two molecular markers. The microsatellite evidence showed signs of a past bottleneck, yet relatively high levels of genetic diversity and low genetic differentiation between two sampling sites. In contrast, the mtDNA revealed moderate to low levels of diversity between sampling sites, and strong genetic differentiation. An explanation for the conflicting patterns may be that the current genetic signature, as depicted by the microsatellite data, is not yet reflected in the mitochondrial dataset; and, as such the data are depicting a timeline for genetic variation within the WLT. Both markers revealed important information about the two sampling sites, which can help inform conservation management of the species.
\end{abstract}

Key words.-Africa; Amietophrynus; Bufonidae; conservation; endangered; genetic variation.

\section{INTRODUCTION}

Intraspecific genetic diversity provides the basis for evolutionary change and is therefore considered the most fundamental level of biodiversity (May 1994). This diversity is governed by the loss and gain of alleles, which can be the result of mutations, random genetic drift, natural and sexual selection or migration. In our human-mediated world, ecological

*Corresponding author. Email: jessica.m.dasilva@gmail.com 
disturbance is proving to be a key driver of changes to the effective population size $\left(N_{\mathrm{e}}\right)$ of a species (for an in-depth review, see Banks et al. 2013); and, over time, these changes can greatly affect a species' genetic diversity (Alcala \& Vuilleumier 2014; Ellegren \& Galtier 2016). It is well established that a reduction in genetic diversity compromises the adaptive potential of a species and, therefore, decreases its long-term survival (Lacy 1997; Markert et al. 2010). This is especially true for threatened species, which can sometimes be characterised by low levels of genetic variation, often due to inbreeding, population bottlenecks, or the disproportionate effects of genetic drift on small populations (e.g. England et al. 2003; Spielman et al. 2004; Allendorf 2005; Willoughby et al. 2015).

Molecular markers, such as mitochondrial DNA (mtDNA) and microsatellite loci, are powerful tools for estimating the diversity among individuals and populations (e.g. Avise et al. 1987; Schwartz et al. 2007). However, patterns of genetic variation are not always congruent between the two. At times, greater population differentiation has been found using microsatellite markers (e.g. Lu et al. 2001; Johnson et al. 2003; De Oliveira Francisco et al. 2013; Kolleck et al. 2013; da Silva et al. 2016); while the reverse has also been documented (e.g. Castella et al. 2001; Yang \& Kenagy 2009). Some possible explanations for these discrepancies include: (i) differences in the selection intensities acting on each marker; (ii) the different mutation rates between markers; and, (iii) the $N_{\mathrm{e}}$ for maternally-inherited markers, such as mtDNA, differ from those of microsatellites which are biparentally inherited markers (Johnson et al. 2003). Genetic diversity is also influenced by patterns of mating, sex-biased dispersal and other demographic parameters (Chesser \& Baker 1996; Johnson et al. 2003; Yang \& Kenagy 2009; De Oliveira Francisco et al. 2013; Kolleck et al. 2013), which can further contribute to the discrepancies in genetic diversity estimated between mtDNA and microsatellite markers. In general, mtDNA is most often used to analyse phylogeographic events, while microsatellites typically provide more fine-scale resolution of more recent demographic events (e.g. Avise et al. 1987). Consequently, researchers must carefully determine what questions they want to address, so that the appropriate markers can be utilised (Hoban et al. 2013). This is especially critical for research on threatened species that have small and/or declining populations, such as the Western Leopard Toad (WLT: Sclerophrys pantherina; previously Bufo pantherina and Amietophrynus pantherinus).

The WLT is endemic to the winter rainfall region of the southwestern Cape of South Africa where it falls into two historically disjunct areas: (1) to the west of False Bay, including the Cape metropolitan area, and (2) to the east of False Bay (De Villiers 2004; Measey \& Tolley 2011; Fig. 1). Its Endangered status is based on its small distribution (area of occupancy [AOO] $440 \mathrm{~km}^{2}$ ) and the ongoing reduction in quantity and quality of habitat associated with urbanisation and agricultural expansion throughout its range (de Villiers 2004; SA-FRoG 2016; Measey 2011). Adults are explosive breeders, moving to breeding sites during the antipodean winter (late July to early September) where they lay large clutches of spawn in strings (Cherry 1992b; de Villiers 2004). Tadpoles take around three months to reach metamorphosis, and males are known to breed after one year and females after two (Cherry 1992a).

In order to investigate the structure and distribution of $S$. pantherina across its range, a recent study utilised the mitochondrial marker, ND2 (Measey \& Tolley 2011), as it was thought to have sufficient population-level variation (Cunningham \& Cherry 2004). Two genetically distinct populations were recognised that corresponded to the distribution of the toads (refer to Fig. 1): population 1 - toads within the Cape Metropolitan area 


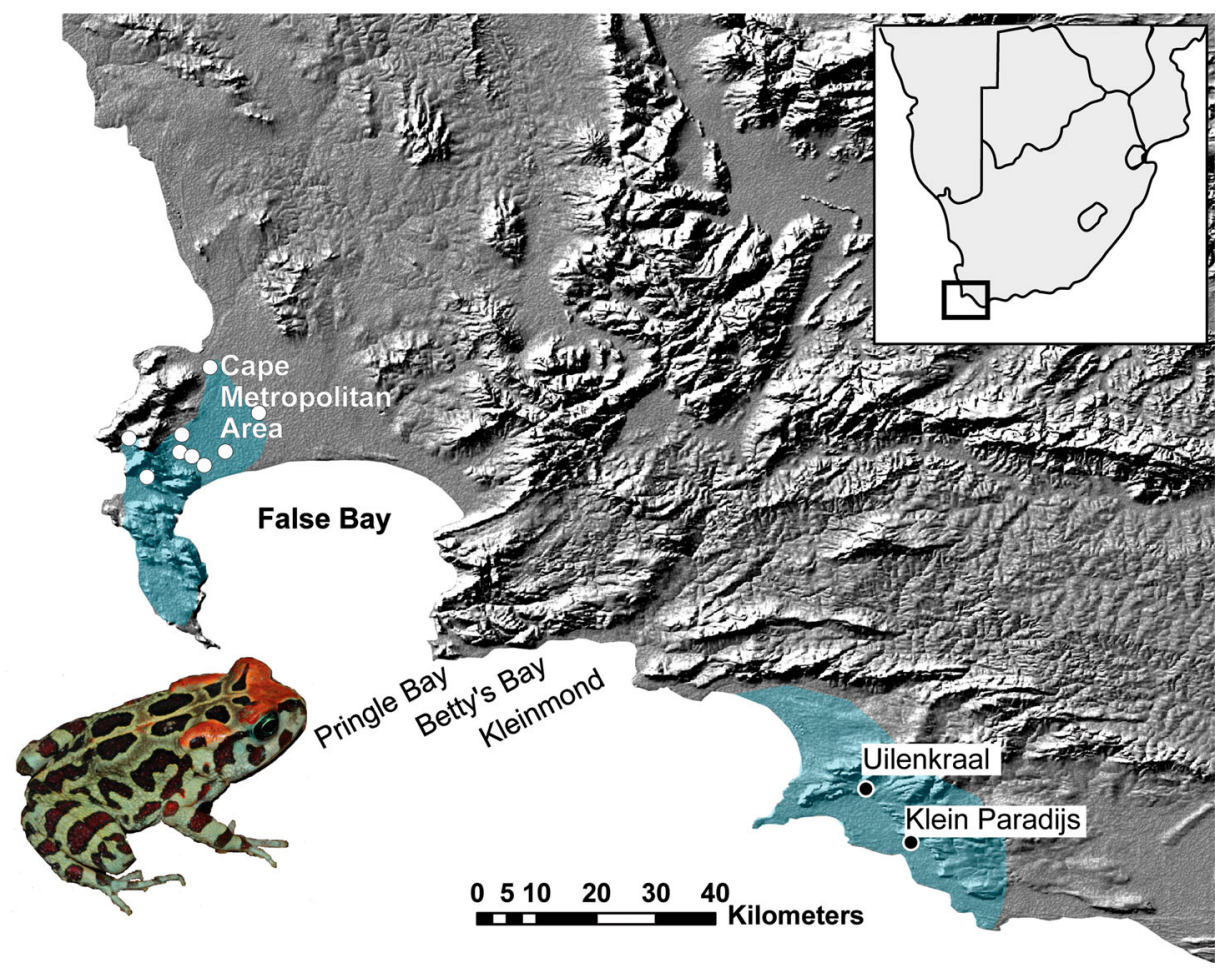

Figure 1. Map depicting the distribution of Sclerophrys pantherina (inset) in the southwestern Cape, South Africa. Shaded blue areas show the disjunct distribution of the species. Circles denote known sampling sites: black-filled circles denote the two sampling sites included in this study; while the white filled circles are sampling sites within the Cape Metropolitan Area (refer to Measey \& Tolley, 2011).

(CMA) - and population 2 - toads east of False Bay (EFB). Moreover, haplotype and nucleotide diversity were greater for the CMA population compared to the EFB population, with no shared haplotypes across the two populations (Measey \& Tolley 2011).

The relatively low genetic diversity found among EFB individuals, coupled with local extinctions and a small number of remaining breeding sites could be an indication that this population is more vulnerable than the CMA population (Measey \& Tolley 2011). With ongoing development in the EFB lowlands due to agriculture and tourism, the vulnerability of this population is likely to worsen. Consequently, it has been recommended that this population be treated as a separate management unit to safeguard the future of the remaining breeding sites (Measey \& Tolley 2011).

Given that this previous study only utilised a single mtDNA marker and the two populations were estimated to have diverged as recently as 1.2 thousand years ago (Measey \& Tolley 2011), the results may not adequately reflect the current genetic diversity within the species. Consequently, in this study, we developed and analysed novel microsatellite markers for $S$. pantherina and compared the results to the previously published ND2 sequence data, specifically for the EFB population, to compare the level of genetic diversity between the two molecular markers. 


\section{Materials ANd Methods}

\section{Data Collection}

Sampling was conducted during the 2010 breeding season in July and August at night when the toads are most active. Two sampling sites within the EFB were targeted: Uilenk-

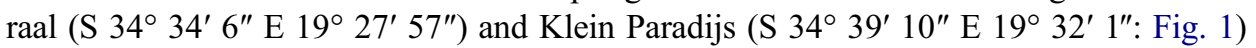
which are $11.25 \mathrm{~km}$ apart in a straight line. Both sites are artificial impoundments which S. pantherina have used for oviposition for many years. A single toe clip (the tip to the first articulation on the inside toe on the left foot) was collected for each toad using sterilised surgical scissors. In addition to providing tissue for DNA analysis, the toe clip was used as an identification of previously captured individuals. Each toe clip was stored in $99 \%$ ethanol and stored at $-40{ }^{\circ} \mathrm{C}$ until DNA was extracted.

\section{Microsatellite Development and Optimisation}

Twelve microsatellite markers were developed for $S$. pantherina (online supplemental material, Table S1), using an enrichment protocol (Glenn \& Schable 2005). Genomic DNA (gDNA) from one individual was digested with RsaI and XmnI, and SuperSNX24 linkers were ligated onto the ends of gDNA fragments. Linkers act as priming sites for polymerase chain reactions $(\mathrm{PCR})$ in subsequent steps. Five tetranucleotide $\left[(\mathrm{AAAT})_{8}\right.$, $\left.(\mathrm{AACT})_{8},(\mathrm{AAGT})_{8},(\mathrm{ACAT})_{8},(\mathrm{AGAT})_{8}\right]$ and six trinucleotide $\left[(\mathrm{AAT})_{12},(\mathrm{ACT})_{12}\right.$, $\left.(\mathrm{AGC})_{6},(\mathrm{AAG})_{8},(\mathrm{AAC})_{6}(\mathrm{ATC})_{8}\right]$ biotinylated probes were hybridised to gDNA in two separate reactions. Streptavidin-coated magnetic beads (Dynabeads ${ }^{\circledR}$ M-280 Invitrogen, Carlsbad, CA) were added to the probe-gDNA complexes, and this mixture was washed twice with $2 \times \mathrm{SSC}, 0.1 \% \mathrm{SDS}$ and four times with $2 \times \mathrm{SSC}, 0.1 \% \mathrm{SDS}$ at $52{ }^{\circ} \mathrm{C}$. Between washes, a magnetic particle collecting unit was used to capture the magnetic beads which are bound to the biotin-gDNA complex. This allows us to capture gDNA containing repeats while other fragments are washed away. Enriched fragments were removed from the probes by denaturing at $95{ }^{\circ} \mathrm{C}$ and precipitated with $3 \mathrm{M}$ sodium acetate and $95 \%$ ethanol. To increase the amount of fragments, a "recovery" PCR was performed in a $25 \mu 1$ reaction containing $1 \times$ PCR buffer $(10 \mathrm{~mm}$ Tris- $\mathrm{HCl}, 50 \mathrm{mM} \mathrm{KCl}, \mathrm{pH} 8.3), 1.5 \mathrm{mM} \mathrm{MgCl}_{2}$, $0.16 \mathrm{~mm}$ of each dNTP, $0.52 \mu \mathrm{M}$ of the SuperSNX24 forward primer, 10 $\times$ BSA, 1U Taq DNA polymerase, and approximately $25 \mathrm{ng}$ enriched gDNA fragments. Thermal cycling, performed in a BIO-RAD DYAD, was as follows: $95{ }^{\circ} \mathrm{C}$ for $2 \mathrm{~min}$ followed by 25 cycles of $95{ }^{\circ} \mathrm{C}$ for $20 \mathrm{~s}, 60^{\circ} \mathrm{C}$ for $20 \mathrm{~s}$ and $72{ }^{\circ} \mathrm{C}$ for $90 \mathrm{~s}$, and a final elongation step of $72{ }^{\circ} \mathrm{C}$ for $30 \mathrm{~min}$. PCR fragments were then cloned using the TOPO-TA Cloning ${ }^{\circledR}$ kit following the manufacturer's protocol (Invitrogen). Bacterial colonies containing a vector with gDNA (i.e. white colonies) were used as a template for subsequent PCR. PCR products were then cleaned using Shrimp Alkaline Phosphatase and Exonuclease I according to the manufacturer's protocol (Affymetrix, Santa Clara, CA). DNA sequencing was performed using the BigDye ${ }^{\circledR}$ Terminator v3.1 Cycle Sequencing Kit (Applied Biosystems, Foster City, CA). Sequencing reactions were precipitated with $125 \mathrm{mM}$ EDTA and $100 \%$ ethanol and run on an ABI 3730 DNA Analyzer. Primer3 (http://frodo.wi. mit.edu/cgi-bin/primer3/primer3_www.cgi) was used to develop microsatellite PCR primers.

Optimisation of the 12 markers was carried out in a $10 \mu \mathrm{l}$ reaction volume containing approximately 5-50 ng of DNA template, $80 \mu \mathrm{M}$ of dNTPs, and $0.2 \mu \mathrm{M}$ of each primer. 
The PCR buffer concentrations, $\mathrm{MgCl}_{2}$ concentrations and annealing temperatures $\left(T_{\mathrm{a}}\right)$ varied with the Taq used and the loci targeted (Table S1). Thermal cycling parameters were as follows: $95{ }^{\circ} \mathrm{C}$ for $4 \mathrm{~min}$, followed by 40 cycles at $95{ }^{\circ} \mathrm{C}$ for $30 \mathrm{~s}, T_{\mathrm{a}}$ for $30 \mathrm{~s}$, $72{ }^{\circ} \mathrm{C}$ for $45 \mathrm{~s}$ and a final extension at $72{ }^{\circ} \mathrm{C}$ for $5-10 \mathrm{~min}$. Successful products were then combined in a poolplex format and profiled at the Central Analytical Facility at Stellenbosch University using an ABI 3100xl Prism (Applied Biosystems, Foster City, CA), ROX 500 or LIZ 500 as the internal size standard, and POP-7 as the polymer, as per manufacturer's recommendation. Alleles were scored and binned using the microsatellite plugin in Geneious version 9.1.5 (Kearse et al. 2012).

\section{Data Analysis}

Microsatellite genotypes.-Microsatellites were screened for genotyping errors and null alleles using Micro-Checker version 2.2.3 (Van Oosterhout et al. 2004) as loci possessing either of these would not typically be considered useful in population-level studies as they may affect the estimation of population differentiation, for example, by reducing the genetic diversity within populations (e.g. Paetkau \& Strobeck 1995). As such, some studies have attempted to correct for null alleles in population genetic studies by statistically adjusting the visible allele and genotype frequencies (e.g. Roques et al. 1999; Chapuis \& Estoup 2007). However, the estimation of null alleles can be biased upwards in populations that are either inbred or consist of closely related individuals (Chybicki \& Burczyk 2009; Campagne et al. 2012), and, conversely, that estimations of inbreeding can be biased upwards when null alleles or genotyping errors occur (e.g. Björklund 2005). Consequently, automatically correcting for null alleles might be unnecessary and inappropriate. To better assess the likely presence of null alleles, we conducted a simultaneous estimation of the inbreeding coefficient (f), null allele frequencies (n), and random genotyping failure (b) using the software INEst v2 (Chybicki et al. 2011). This method uses a Bayesian approach to simultaneously estimate the three parameters, which should provide a more accurate estimation of each parameter because it allows for the relative contribution of each through selection of the best fitting model (i.e. model with the lowest Deviance Information Criterion [DIC]).

The Markov Chain Monte Carlo (MCMC) was run with 500000 cycles and $10 \%$ burn-in, saving parameters every 100 cycles. The DICs from each model were then compared, with the better (lowest) scoring model considered a better fit. Given that the effects of null alleles are locus-specific (Dakin \& Avise 2004), their presence should be reflected in the best fitting models of each population, or in this case, sampling site. An examination of the $p[j, k]$ parameter from the INEst results should provide confirmation of the locus/ loci containing null alleles This parameter examines the frequency of the $k$ th allele at the $j$ th locus, where $p[j, 0]$ denotes the null allele frequency at the $j$ th locus (Chybicki et al. 2011), For these loci, we manually adjusted allele frequencies using the Brookfield 1 algorithm implemented in Micro-Checker. This method discounts nonamplifying individuals when calculating null allele frequencies, as opposed to the Brookfield 2 algorithm, which treats non-amplifications as data and regards them as null homozygotes when calculating null allele frequencies (Brookfield 1996). By correcting for null alleles in this way, we were able to maximise sample size and retain loci that may have sufficient variability to detect patterns. All subsequent analyses were conducted on this corrected dataset. 
Genepop on the Web (Raymond \& Rousset 1995; Rousset 2008) was used to test for linkage disequilibrium (LD) between loci using the log-likelihood ratio test (Cockerham \& Weir 1977). Analyses for LD were run with a MCMC dememorisation of 10000 for 100 batch runs of 1000 iterations. For each locus, the number of alleles (allelic richness: $A_{\mathrm{R}}$ ), allelic size range, observed $\left(H_{\mathrm{O}}\right)$ and expected $\left(H_{\mathrm{E}}\right)$ heterozygosities (Nei 1987), and deviations from Hardy-Weinberg equilibrium (HWE) were also estimated for the two sampling sites using ARLEQUIN version 3.5.1.2 (Excoffier \& Lischer 2010). Deviations from HWE were tested using $1.0 \times 10^{6}$ Markov chains and 100000 dememorisation steps. To minimise the possibility of Type I errors, tests for linkage and Hardy-Weinberg disequilibria were corrected for multiple comparisons by applying Holm's sequential Bonferroni correction (Holm 1979; Rice 1989).

ARLEQUIN was used to test for signatures of genetic bottlenecks in the two sampling sites using the Garza-Williamson $M$-ratio (G-W: Garza \& Williamson 2001) and to determine the level of gene flow between sites using $R_{\mathrm{ST}}$ (Slatkin 1995). The $M$-ratio is estimated according to the equation $M=\mathrm{k} / \mathrm{R}+1$, where k represents the number of alleles at a locus and $\mathrm{R}$ is the associated allelic range (Garza \& Williamson 2001; Excoffier \& Lischer 2010). Populations that have experienced a reduction in their effective population size exhibit a larger reduction in allele numbers than range (Excoffier \& Lischer 2010). Accordingly, an $M$-ratio less than 0.68 (value derived from stable wild populations) would indicate that the sampling site has been through a bottleneck at the locus under examination, whereas a value closer to one is indicative of stationary/stable populations (Garza \& Williamson 2001; Peery et al. 2012). Gene flow was measured using $R_{\mathrm{ST}}$ instead of $F_{\mathrm{ST}}$ because it relies on a stepwise mutation model which is better suited for the high mutation rates and memory dependent allele mutations found within microsatellite loci (Di Rienzo et al. 1994; Slatkin 1995). In contrast, $F_{\mathrm{ST}}$ relies upon the infinite allele model, which assumes low mutation rates and a mutation process independent of the prior allelic state (Weber \& Wong 1993; Slatkin 1995). The number of migrants $\left(N_{\mathrm{m}}\right)$ between populations was then calculated from the equation, $N_{\mathrm{m}}=0.25^{*}\left(1-F_{\mathrm{ST}}\right) / F_{\mathrm{ST}}$ (Slatkin \& Barton 1989), using $R_{\mathrm{ST}}$ in place of $F_{\mathrm{ST}}$. Genetic differentiation can be categorised as great if $R_{\mathrm{ST}}>0.15$, moderate if $R_{\mathrm{ST}}=0.05-0.15$, and little if $R_{\mathrm{ST}}<0.05$ (Wright 1978). Similarly, the level of gene flow between sampling sites is categorised as high if $N_{\mathrm{m}}>1$, intermediate if $N_{\mathrm{m}}=0.25$ to 0.99 , and low if $N_{\mathrm{m}}<0.25$ (Wright 1978).

mtDNA sequences.-The ND2 sequence data for Uilenkraal and Klein Paradijs data from Measey \& Tolley (2011) was used to estimate genetic diversity within S. pantherina. Specifically, the number of haplotypes $(h)$, haplotypic diversity $(h d)$, nucleotide diversity $(\pi)$, the number of polymorphic sites $(S)$, and the total number of mutations $(E t a)$ were calculated using DnaSP version 5.10.1 (Librado \& Rozas, 2009). Haplotype diversity (or gene diversity) is defined as the probability that two randomly sampled alleles are different; and, nucleotide diversity is defined as the average number of nucleotide differences per site in pairwise comparisons among DNA sequences (Nei 1987). DnaSP was also used to determine the level of genetic differentiation and gene flow between sampling sites; and, $\Phi_{\mathrm{ST}}$, which is analogous to $F_{\mathrm{ST}}$, was calculated in ARLEQUIN. $F_{\mathrm{ST}}$ is an allelic calculation that assumes all alleles are equidistant from each other; whereas $\Phi_{\mathrm{ST}}$ (the nucleotide diversity calculation) allows for different distances between different alleles. Lastly, a medianjoining network was created using NETWORK, version 5.0 (Bandelt et al. 1999) to visualise the relationships among the mtDNA haplotypes between the two sampling sites. 


\section{Results}

\section{Microsatellite Variation}

All 12 loci were polymorphic with the number of alleles ranging from 3 to 18 (Table 1). There was no evidence of large allele dropout or scoring errors due to stutter peaks; however, the likely presence of null alleles due to homozygote excess was detected in both Klein Paradijs and Uilenkraal sampling sites (Tables 1 and 2). Because null alleles are locus-specific, WLT16 and WLT39 likely represent 'true' null alleles. Although the best-fitting INEst models also indicated the influence of inbreeding, with average $F_{\text {IS }}=0.0247$ for Uilenkraal and 0.0588 for Klein Paradijs sampling sites, the effects are minimal. An examination of the $95 \%$ posterior probability intervals for $F_{\text {IS }}$ included zero for Uilenkraal, indicating that there is no significant inbreeding in this population (Table 2).

No significant linkage disequilibrium was detected for any of the loci; however, two loci were found to deviate from HWE (WLT39 \& WLT42: Table 1). Because locus WLT39 was identified as possibly having null alleles across populations, this is the most probable explanation for its deviation from HWE. Locus WLT42, on the other hand, only showed deviations from HWE for Klein Paradijs, indicating that these deviations are most likely attributed to some biological factor such as the Wahlund effect or inbreeding (Chakraborty et al. 1992). Given the highly fragmented nature of the WLT habitat, it is possible that small populations have become fixed for diverse alleles.

Evidence of a genetic bottleneck was found across all loci for both sampling sites ( $M$-ratio: Table 1), which also show fairly similar levels of microsatellite genetic diversity for all indices examined (Table 3$)$ and exhibit little differentiation $\left(R_{\mathrm{ST}}: 0.082, P<0.001 ; N_{\mathrm{m}}: 2.79\right)$.

\section{mtDNA Variation}

Unlike the microsatellite results, analysis of the mtDNA data revealed differing levels of diversity between Uilenkraal and Klein Paradijs sampling sites (Table 3). In particular, Uilenkraal was found to have moderate haplotype diversity ( $h d=0.567 \pm 0.051$ ), yet low nucleotide diversity $(\pi=0.00095 \pm 0.00022)$; while Klein Paradijs has low haplotype diversity ( $h d=0.154 \pm 0.126)$ and very low nucleotide diversity $(\pi=0.00019 \pm 0.0016)$. The two populations share one haplotype, possessed by the majority of individuals sampled (Fig. 2), indicating a previously continuous population or past gene flow between populations; however, the two populations were found to be strongly differentiated $\left(\Phi_{\mathrm{ST}}=0.41991, P=0.002\right)$.

\section{Discussion}

Although the mtDNA and microsatellite evidence presented here reveal different patterns with respect to the genetic diversity within the two sampling sites of $S$. pantherina, similarities were also present with both markers showing signs of population bottlenecks and the presence of gene flow between Uilenkraal and Klein Paradijs. A possible explanation for the conflicting patterns of genetic diversity may be that the current genetic signature (as depicted by the microsatellite data) is not yet reflected in the mitochondrial dataset due to the slower evolution of the mtDNA markers. The data may, therefore, be depicting a timeline for genetic variation within the WLT, with the mtDNA data revealing the more 
Table 1. Descriptive statistics of genetic variability for 12 Sclerophrys pantherina microsatellite loci across two sampling sites in the southwestern Cape, South Africa.

\begin{tabular}{|c|c|c|c|c|c|c|c|c|c|c|c|c|c|c|}
\hline \multirow{2}{*}{ Locus } & \multicolumn{7}{|c|}{ Uilenkraal ( $n=39)$} & \multicolumn{7}{|c|}{ Klein Paradijs $(n=41)$} \\
\hline & $N_{\mathrm{A}}$ & $S$ & $H_{\mathrm{O}}$ & $H_{\mathrm{E}}$ & HWE & Null & $M$ & $N_{\mathrm{A}}$ & $S$ & $H_{\mathrm{O}}$ & $H_{\mathrm{E}}$ & HWE & Null & $M$ \\
\hline WLT2 & 13 & 119-179 & 0.81 & 0.88 & 0.2772 & No & 0.230 & 10 & $123-167$ & 0.80 & 0.80 & 0.5150 & No & 0.222 \\
\hline WLT16 & 14 & $142-358$ & 0.62 & 0.92 & 0.0006 & Maybe & 0.065 & 15 & $142-358$ & 0.74 & 0.91 & 0.0411 & Maybe & 0.069 \\
\hline WLT25 & 11 & $167-219$ & 0.92 & 0.90 & 0.8701 & No & 0.226 & 9 & $159-207$ & 0.69 & 0.81 & 0.0443 & No & 0.204 \\
\hline WLT36 & 14 & $216-276$ & 0.90 & 0.89 & 0.6666 & No & 0.230 & 9 & $216-280$ & 0.75 & 0.82 & 0.1783 & No & 0.138 \\
\hline WLT40 & 10 & $152-200$ & 0.61 & 0.85 & 0.0054 & Maybe & 0.204 & 9 & $148-200$ & 0.71 & 0.85 & 0.0643 & No & 0.170 \\
\hline WLT42 & 6 & 179-211 & 0.39 & 0.43 & 0.1636 & No & 0.182 & 5 & $187-211$ & 0.27 & 0.60 & $<0.001^{*}$ & Maybe & 0.240 \\
\hline WLT44 & 15 & $195-347$ & 0.74 & 0.87 & 0.0260 & Maybe & 0.118 & 8 & $195-343$ & 0.75 & 0.75 & 0.4391 & No & 0.054 \\
\hline WLT76 & 4 & $174-183$ & 0.57 & 0.54 & 0.8948 & No & 0.400 & 4 & $174-183$ & 0.73 & 0.67 & 0.5540 & No & 0.400 \\
\hline WLT88 & 7 & $197-245$ & 0.67 & 0.74 & 0.7256 & No & 0.163 & 6 & $197-245$ & 0.63 & 0.78 & 0.0237 & Maybe & 0.142 \\
\hline
\end{tabular}

$N_{\mathrm{A}}$, Number of observed alleles; $S$, allele size range; $H_{\mathrm{O}}$, observed and $H_{\mathrm{E}}$, expected heterozygosity; HWE, Hardy-Weinberg equilibrium $P$ value; Null, presence of null alleles; $M$, Garza-Williamson index. Significant deviations from HWE are denoted by *, based on a Bonferroni significance value of $0.0042(P<0.05 / 12)$. 
Table 2. Results of Bayesian individual inbreeding model for Sclerophrys pantherina. Models were run using all 12 microsatellite loci. Model parameters include inbreeding (f), null alleles (n) and random genotyping errors (b).

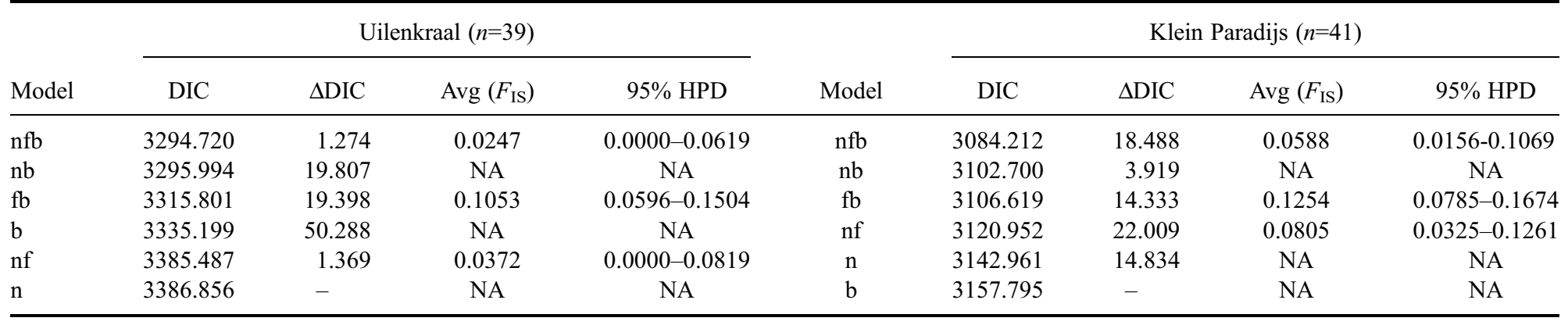

DIC, Deviance Information Criterion; $\triangle \mathrm{DIC}$, difference in DIC from the best model; Avg $\left(F_{\mathrm{IS}}\right)$, average inbreeding coefficient for the population; HPD, highest probability density. 
Table 3. Mean microsatellite and mitochondrial DNA genetic diversity indices for Sclerophrys pantherina from two sampling sites.

\begin{tabular}{lcc}
\hline Genetic diversity & Uilenkraal & Klein Paradijs \\
\hline Microsatellites & & \\
Sample size $(n)$ & 39 & 41 \\
Allelic richness $\left(A_{\mathrm{R}}\right)$ & 10.667 & 8.911 \\
Observed heterozygosity $\left(H_{\mathrm{O}}\right)$ & 0.693 & 0.667 \\
Expected heterozygosity $\left(H_{\mathrm{E}}\right)$ & 0.795 & 0.770 \\
$m t D N A$ & & \\
Sample size $(n)$ & 22 & 13 \\
Length $($ bp) & 741 & 741 \\
No. haplotypes $(h)$ & 3 & 2 \\
Haplotypic diversity $(h d)$ & $0.567 \pm 0.051$ & $0.154 \pm 0.126$ \\
Nucleotide diversity $(\pi)$ & $0.00095 \pm 0.00022$ & $0.00019 \pm 0.00016$ \\
No. polymorphic sites $(S)$ & 3 & 1 \\
Total no. mutations $(E t a)$ & 3 & 1 \\
\hline
\end{tabular}

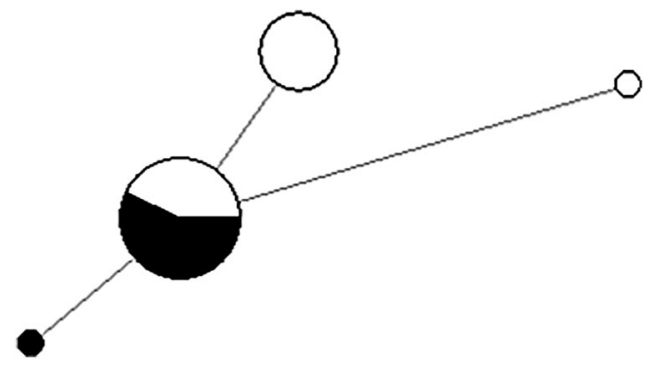

Figure 2. A median-joining haplotype network based on the ND2 mitochondrial gene for Sclerophrys pantherina. Haplotypes are represented by circles and show the proportion of toads from Uilenkraal (white; $n=22$ ) and Klein Paradijs (black; $n=13$ ). The area of the circle is proportional to the number of individuals with that haplotype, and the length of the connecting lines is proportional to the number of base changes between haplotypes (refer to Table 3).

ancestral relationships and the microsatellite data the most recent demographic structuring. Both markers revealed important information about the two sampling sites, which can help inform conservation management of the species.

The mtDNA data uncovered different levels of diversity between Uilenkraal and Klein Paradijs, with the former showing moderate haplotype diversity and low nucleotide diversity, and the latter showing low levels of both diversity measures. The low nucleotide diversity estimates suggest that both populations underwent a bottleneck in the recent past. There may have been a single WLT population in the EFB region, or possibly even across the entire range of $S$. pantherina, during the last glacial maxima when areas of the continental shelf were exposed by lowered sea levels (Schreiner et al. 2013; Mokhatla et al. 2015). Then, during the Holocene, the rising sea levels and a distinct drying period may have caused a significant retraction in the distribution of the species, creating a population bottleneck (Measey \& Tolley, 2011), and through time differentiation between the two sampling sites.

Evidence of a bottleneck is also reflected in the microsatellite results; however, the data also indicate strong gene flow between sites and moderate to high levels of overall genetic variation as indicated by the allelic richness (the most robust indicator for monitoring 
genetic diversity and decline: Hoban et al. 2014) of each sampling site $\left(A_{R}=10.7\right.$ and 8.9 for Uilenkraal and Klein Paradijs, respectively; Table 3), compared to other toad species (e.g. Wu \& Hu 2010; Roth \& Jehle 2016; da Silva et al. 2016). These microsatellite data tend to depict the more recent genetic diversity for the species; and, on the surface, it could be interpreted that the two sampling sites are now healthy and thriving after the bottleneck. However, high levels of genetic diversity do not always equate to viable populations (Habel \& Schmitt 2012).

Levels of genetic diversity differ greatly among species and especially between ecological generalists and specialists. Generalist species tend to have high genetic diversity within their populations and low genetic differentiation among them due to the absence of bottlenecks, strong gene flow, and little effects of genetic drift. Specialist species tend towards low genetic diversity and strong genetic differentiation due to small or fluctuating populations that experience bottlenecks (e.g. Hughes et al. 1999; Schmitt et al. 2005; Habel \& Schmitt 2009, 2012). Based on these distinctions, S. pantherina is probably not a true habitat specialist nor generalist, but might be an ecologically intermediate species. Such species must maintain high levels of diversity within their populations, which is facilitated by gene flow, thereby forcing them to exist in metapopulations (Habel \& Schmitt 2012). Without a network of populations, the species might suffer greatly from the consequences of population bottlenecks, which result in shifts in allele frequencies, often resulting in the loss of genetic adaptations (Luikart et al. 1998).

Unlike many of the Cape lowland endemics (e.g. Xenopus gilli, Microbatrachella capensis), the WLT appears to have a high tolerance for disturbance with most, if not all, of its populations currently breeding in anthropogenically altered habitats (Mokhatla et al. 2015). This adaptability will be essential to the long-term survival of this species, which will need to counter predicted fragmentation due to newly created impoundments in both urban and rural areas (Mokhatla et al. 2015). In addition, this species faces the threat of an invasive congener currently spreading in the CMA range (Measey et al. 2017). However, there might be a threshold of disturbance beyond which populations are not able to recover. This might very well explain the loss of other sampling sites in the EFB region, which were known prior to the 1990s (i.e. Pringle Bay, Betty's Bay and Kleinmond: Measey \& Tolley 2011; Fig. 1).

Given that the EFB is subject to ongoing development, the two sampling sites examined here, especially Klein Paradijs, may also be at risk. It is, therefore, imperative that the current genetic diversity within these two sampling sites be maintained over time, with conservation efforts focused on preserving connectivity between sites to ensure adequate gene flow between sampling sites. Future studies using these novel microsatellites could then be used to monitor the frequency of dispersal between sampling sites and, ultimately, the extent of genetic erosion/preservation within the EFB.

\section{ACKNOWLeDGements}

We would like to thank the South African National Biodiversity Institute and the Field Museum's Pritzker Laboratory for Molecular Systematics and Evolution for providing logistical support and funding for this study. Additional funding was provided by the SANBI-NORAD Threatened Species Programme. Many thanks also to the landowners, who gave permission to access their property, Susanna Fuchs and Aletta Groenwald. Sampling was conducted under permit from the Western Cape provincial conservation authority, CapeNature (\#AAA004-00090-0035) and an ethical clearance certificate (\# 0001/08 from SANBI Ethics Committee). 


\section{SupPlemental Material}

Supplemental data for this article can be accessed at http://dx.doi.org10.1080/21564574. 2017.1294115

\section{REFERENCES}

Alcala, N. \& S. Vuilleumier. 2014. Turnover and accumulation of genetic diversity across large time-scale cycles of isolation and connection of populations. Proc. R. Soc. B. 281: 20141369.

Allendorf, F. 2005. Genetic drift and the loss of alleles versus heterozygosity. Zoo Biol. 5: 181-190.

Avise, J.C., J. Arnold, R.M. Ball, Jr, E. Bermingham, T. Lamb, J.E. Neigel, C.A. Reeb \& N.C. SAUNDERS. 1987. Intraspecific phylogeography: the mitochondrial DNA bridge between population genetics and systematics. An. Rev. Ecol. Syst. 18: 489-522.

Bandelt, H.-J., P. Forster \& A. RöHL. 1999. Median-joining networks for inferring intraspecific phylogenies. Mol. Biol. Evol. 16: 37-48.

Banks, S.C., G. J. Cary, A.L. Smith, I.D. Davies, D.A. Driscoll, A.M. Gill, D. B. Lindenmayer \& R. PeaKall. 2013. How does ecological disturbance influence genetic diversity? Trends Ecol. Evol. 28: 670-679.

BJöRKLUND, M. 2005. A method for adjusting allele frequencies in the case of microsatellite allele drop-out. Mol. Ecol. Notes 5: 676-679.

BrooKfiELD, J.K.Y. 1996. A simple new method for estimating null allele frequency from heterozygote deficiency. Mol. Ecol. 5: 453-455.

Campagne, P., P.E. Smouse, G. Varouchas, J.F. Silvain \& B. Leru. 2012. Comparing the van Oosterhout and Chybicki-Burczyk methods of estimating null allele frequencies for inbred populations. Mol. Ecol. Resour. 12: 975-982.

Castella, V., M. Ruedi \& L. Excoffier. 2001. Contrasted patterns of mitochondrial and nuclear structure among nursery colonies of the Myotis myotis. J. Evol. Biol. 14: 708-720.

Chakraborty, R., M. De Andrade, S. P. Daiger \& B. Budowle. 1992. Apparent heterozygote deficiencies observed in DNA typing data and their implications in forensic applications. Ann. Hum. Genet. 56: 45-57.

Chapuis, M.-P. \& A. Estoup. 2007. Microsatellite null alleles and estimation of population differentiation. Mol. Biol. Evol. 24: 621-631.

Cherry, M.I. 1992a. Body size, age and reproduction in the leopard toad, Bufo pardalis. J. Zool. Lond. 228: 41-50.

Cherry, M.I. 1992b. Sexual selection in the leopard toad, Bufo pardalis. Behaviour 120: 164-176.

CHEsser, R.K. \& R.J. BAKer. 1996. Effective sizes and dynamics of uniparentally and diparentally inherited genes. Genetics 144: 1225-1236.

Chybicki, I.J. \& J. BurczyK. 2009. Simultaneous estimation of null alleles and inbreeding coefficients. J. Hered. 100: 106-113.

Chybicki, I.J., A. Oleska \& J. Burczyk. 2011. Increased inbreeding and strong kinship structure in Taxus baccata estimated from both AFLP and SSR data. Heredity 107: 589-600.

Cockerham, C.C. \& B.S. WeIR. 1977. Digenic descent measures for finite populations. Genet. Res. 30: 121-147.

Cunningham, M. \& M.I. Cherry. 2004. Molecular systematics of African 20-chromosome toads (Anura: Bufonidae). Mol. Phylogenet. Evol. 320: 671-685.

DAKIN, E.E. \& J.C. AvISE. 2004. Microsatellite null alleles in parentage analysis. Heredity 93: 504-509.

Da Silva, J.M., K.A. Feldheim, R.J. Daniels, S. Edwards \& K.A. Tolley. 2016. Analysis of genetic diversity in Rose's mountain toadlet (Capensibufo rosei) using novel microsatellite markers. Afr. J. Herp. 65: 69-82.

De Oliveira Francisco, F., L.R. Santiago \& M.C. Arias. 2013. Molecular genetic diversity in populations of the stingless bee Plebeia remota: A case study. Genet. Mol. Biol. 36: 118-123.

De Villiers A. 2004. Species account: Bufo pantherinus A. Smith 1828. In L.R. Minter, M. Burger, J.A. Harrison, J. Bishop \& H. BraAck (Eds) Atlas and Red Data Book of the Frogs of South Africa, Lesotho and Swaziland. Smithsonian Institution Press, Washington DC, pp. 71-74. 
Di Rienzo, A., A.C. Peterson, J.C. Garza, A.M. Valdez, M. Slatkin \& N.B. Freimer. 1994. Mutational processes of simple sequence repeat loci in human populations. Proc. Nat. Acad. Sci. USA. 91: 3166-3170.

Ellegren, H. \& N. Galtier. 2016. Determinants of genetic diversity. Nat. Rev. Genet. 17: 422-433.

England, P.R., G.H.R. Osler, L.M. Woodworth, M.E. Montgomery, D.A. Briscoe \& R. Frankham. 2003. Effects of intense versus diffuse population bottlenecks on microsatellite genetic diversity and evolutionary potential. Conserv. Genet. 4: 595-604.

EXCOFFIER, L. \& H. LisCHER. 2010. Arlequin suite ver 3.5: A new series of programs to perform population genetics analyses under Linux and Windows. Mol. Ecol. Resour. 10: 564-567.

Garza, J.C. \& E.G. WiLliamson. 2001. Detection of reduction in population size using data from microsatellite loci. Mol. Ecol. 10: 305-318.

GlenN, T.C. \& N.A. Schable. 2005. Isolating microsatellite DNA loci. In E.A. Zimmer and E.H. Roalson (Eds) Molecular Evolution: Producing the Biochemical Data, Part B. Vol. 395. Methods in Enzymology. Academic Press, Amsterdam, pp. 202-222.

Habel, J.C. \& Schmitt, T. 2009. The genetic consequences of different dispersal behaviours in two lycaenid butterfly species. Bull. Entomol. Res. 99: 513-523.

Habel, J.C. \& T. Schmitt. 2012. The burden of genetic diversity. Biol. Conserv. 147: 270-274.

Hoban, S., P. Arntzen, G. Bertorelle, J. Bryja, M. Fernandes, K. Frith, O. Gaggiotti, P. Galbusera, J.A. Godoy, H.C. Hauffe, A.R. Hoelzel, R. Nichols, S. Pérez-Espona, C. Primmer, I.-R.M. Russo, G. Segelbacher, H.R. Siegismund, M. Sihvonen, P. Suögren-Gulve, C. Vernesi, C. Vilà \& M.W. BRuford. 2013. Conservation Genetic Resources for Effective Species Survival (ConGRESS): bridging the divide between conservation research and practice. J. Nat. Conserv. 21: 433-437.

Hoban, S., J.A. Arntzen, M.W. Bruford, J.A. Godoy, A.R. Hoelzel, G. Segelbacher, C. Vila \& G. Bertorelle. 2014. Comparative evaluation of potential indicators and temporal sampling protocols for monitoring genetic erosion. Evol. App. 7: 984-998.

Holm, S. 1979. A simple sequential rejective multiple test procedure. Scand. J. Stat. 6: 65-70.

Hughes, T.P., A.H. Baird, E.A. Dinsdale, N.A. Moltschaniwskyj, M.S. Pratchett, J.E. Tanner \& B.L. WILLIS. 1999. Patterns of recruitment and abundance of corals along the Great Barrier Reef. Nature 397: 59-63.

Johnson, J.A., J.E. ToEPFER \& P.O. DunN. 2003. Contrasting mitochondrial and microsatellite population structure in fragmented populations of greater prairie-chickens. Mol. Ecol. 12: 3335-3347.

Kearse, M., R. Moir, A. Wilson, S. Stones-Havas, M. Cheung, S. Sturrock, S. Buxton, A. Cooper, S. Markowitz, C. Duran, T. Thierer, B. Ashton, P. Mentjies \& A. Drummond. 2012. Geneious Basic: an integrated and extendable desktop software platform for the organization and analysis of sequence data. Bioinformatics 28: 1647-1649. http://www.geneious.com

Kolleck, J., M. YAng, D. ZINNER \& C. Roos. 2013. Genetic diversity in Endangered Guizhou Snubnosed monkeys (Rhinopithecus brelichi): Contrasting results from microsatellite and mitochondrial DNA data. PLoS ONE 8: e73647.

LACY, R.C. 1997. Importance of genetic variation to the viability of mammalian populations. J. Mammal. 78: 329-335.

Librado, P. \& J. Rozas. 2009. DnaSP v5: A software for comprehensive analysis of DNA polymorphism data. Bioinformatics 25: 1451-1452.

Lu, G., D.J. BASLEY \& L. Bernatchez. 2001. Contrasting patterns of mitochondrial DNA and microsatellite introgressive hybridization between lineages of lake whitefish (Coregonus clupeaformis): relevance for speciation. Mol. Ecol. 10: 965-985.

Luikart, G., F.W. Allendorf, J.-M. Cornuet \& W.B. Sherwin. 1998. Distortion of allele frequency distributions provides a test for recent population bottlenecks. J. Hered. 89: 238-247.

Markert, J.A., D.M. Champlin, R. Gutjahr-Gobell, J.S. Grear, A. Kuhn \& T.J. McGreevy. 2010. Population genetic diversity and fitness in multiple environments. BMC Evol. Biol. 10: 205.

MAY, R.M. 1994. Biological diversity: differences between land and sea. Phil. Trans. R. Soc Lond. B. 343: 105-111.

Measey, G.J. 2011. Ensuring a future for South Africa's frogs: a strategy for conservation research. SANBI Biodiversity Series 19. South African National Biodiversity Institute, Pretoria.

Measey, G.J. \& K.A. Tolley. 2011. Investigating the cause of the disjunct distribution of Amietophrynus pantherinus, the Endangered South African western leopard toad. Conserv. Genet. 12: 61-70. 
Measey, J., Davies, S., Vimercati, G., Rebelo, A., Schmidt, W. \& Turner, A.A. 2017. Invasive amphibians in southern Africa: a review of invasion pathways. Bothalia 47(2), a2117. https:// doi.org/10.4102/abc.v47i2.2117

Mokhatla, M.M., D. RöDder \& G.J. Measey. 2015. Assessing the effects of climate change on distributions of Cape Floristic Region amphibians. S. Afr. J. Sci. 111: 1-7.

NeI, M. 1987. Molecular Evolutionary Genetics. Columbia University Press, New York.

PaetKau, D. \& C. Strobeck. 1995. The molecular basis and evolutionary history of a microsatellite null allele in bears. Mol. Ecol. 4: 519-520.

Peery, M.Z., R. Kirby, B.N. Reid, R. Stoelting, E. Doucet-Bëer, S. Robinson, C. VÁsquez-Carrillo, J.N. Pauli \& P.J. Palsbøll. 2012. Reliability of genetic bottleneck tests for detecting recent population declines. Mol. Ecol. 21: 3403-3418.

RAYMOND, M. \& F. Rousset. 1995. GENEPOP (version 1.2): population genetics software for exact tests and ecumenicism. J. Heredity 86: 248-249.

Rice, W.R. 1989. Analyzing tables of statistical tests. Evolution 43: 223-225.

Roques, S., P. Duchesne \& L. Bernatchez. 1999. Potential of microsatellites for individual assignment: the North Atlantic redfish (genus Sebastes) species complex as a case study. Mol. Ecol. 8: $1703-1717$.

Roth, S. \& R. JehLe. 2016. High genetic diversity of common toad (Bufo bufo) populations under strong natural fragmentation on a Northern archipelago. Ecol. Evol. 6: 1626-1636.

RousSET, F. 2008. Genepop'007: a complete reimplementation of the Genepop software for Windows and Linux. Mol. Ecol. Res. 8: 103-106.

SA-FRoG (IUCN SSC Amphibian Specialist Group \& South African Frog Re-assessment Group). 2016. Sclerophrys pantherina. The IUCN Red List of Threatened Species 2016: e.T54723A77159333. http://dx.doi.org/10.2305/IUCN.UK.2016-3.RLTS.T54723A77159333.en. Downloaded on 10 January 2017.

Schmitt, T., Röber, S., Seitz, A.. 2005. Is the last glaciation the only relevant event for the present genetic population structure of the Meadow Brown butterfly Maniola jurtina (Lepidoptera: Nymphalidae)? Biol. J. Linn. Soc. 85: 419-431.

SchreInER, C., D. RÖDDER \& G.J. MEASEY. 2013. Using models to test Poynton's predictions. Afr. J. Herpetol. 62: 49-62.

Schwartz, M.K., G. Luikart \& R.S. Waples. 2007 Genetic monitoring as a promising tool for conservation and management. Trends Ecol. Evol. 22: 25-33.

Slatkin, M. 1995. A measure of population subdivision based on microsatellite allele frequencies. Genetics 139: 457-462.

SLATKIN, P.E. \& N.H. BARTON. 1989. A comparison of three indirect methods for estimating average levels of gene flow. Evolution 43: 1349-1368.

Spielman, D., B.W. Brook \& R. Frankham. 2004. Most species are not driven to extinction before genetic factors impact them. Proc. Natl. Acad. Sci. 101: 15261-15264.

Van Oosterhout, C., W.F. Hutchinson, D.P.M. Wills \& P. Shipley. 2004. Micro-Checker: software for identifying and correcting genotyping errors in microsatellite data. Mol. Ecol. Notes 4: 535-538.

Weber, J.L. \& C. Wong. 1993. Mutation of human short tandem repeats. Hum. Mol. Genet. 2: $1123-1128$.

Willoughby, J.R., M. Sundaram, B.K. Wijayawardena, S.J.A. Kimble, Y. Ji, N.B. Fernandez, J.D. Antonides, M.C. Lamb, N.J. Marra \& J.A. DeWoody. 2015. The reduction of genetic diversity in threatened vertebrates and new recommendations regarding IUCN conservation rankings. Biol. Conserv. 191: 495-503.

Wright, S. 1978. Evolution and the Genetics of Populations. Variability Within and Among Natural Populations, Vol. IV. University of Chicago Press, Chicago, IL.

Wu, X.-B., Hu, Y.-L.. 2010. Genetic diversity and molecular differentiation of Chinese toad based on microsatellite markers. Mol. Biol. Rep. 37: 2379-2386.

YANG, D.-S. \& G.J. KeNAGY. 2009. Nuclear and mitochondrial DNA reveal contrasting evolutionary processes in populations of deer mice (Peromyscus maniculatus). Mol. Ecol. 18: 5115-5125. 